\title{
RECENT DEVELOPMENT IN SOLAR WIND MODELING
}

\author{
RUTH ESSER \\ Harvard-Smithsonian Center for Astrophysics, Cambridge, $M A$
}

December 4, 1996

\begin{abstract}
.
An improved knowledge of the physical conditions in the low corona and solar wind can only be obtained through careful comparisons between theoretical descriptions of the solar wind expansion and plasma parameters derived from observations. In this review we will present a summary of recent approaches in solar wind modeling. The plasma parameters characterizing the solar wind models will be compared to constraints inferred from in-situ and remote observations. We will then discuss the implications of the results obtained from this study for future model studies and observations. Emphasis will be placed on high-speed solar wind streams originating from large coronal holes.
\end{abstract}

Key words: Transition Region - Corona - Solar Wind

\section{Introduction}

More than three decades ago Parker $[1958,1963]$ proved that the pressure gradient force can drive charged particles from the gravitationally bound, static solar atmosphere to a supersonic flow. He also established how the flow speed at $1 \mathrm{AU}$ depends on the temperature in the inner corona, and that higher-speed winds most likely require a nonthermal energy flux which might be transported by hydromagnetic waves. Leer and Holzer [1991] and Bürgi [1992] showed that the temperature in the inner corona should be fairly constant for the solar wind mass flux to fall into the small range of mass fluxes observed at $1 \mathrm{AU}$; otherwise some physical processes should exist that regulate the mass flux, such as a significant helium abundance in the inner corona.

Due to our limited knowledge of the physical conditions in the inner corona it has so far not been possible to identify the mechanisms that heat the solar plasma from transition region to coronal temperatures. Therefore most solar wind models have in the past ignored the heating in the inner corona altogether, placing the inner boundary at the temperature maximum, assuming that no heating occurs beyond that boundary. The earliest models that treated the transition region, corona and solar wind as a unity were the models by Hollweg [1986], Hollweg and Johnson [1988], and Withbroe [1988]. Using a one-fluid and two-fluid solar wind model respectively, Hollweg [1986] and Hollweg and Johnson [1988] showed that it is extremely difficult to produce high flow speeds at $1 \mathrm{AU}$ if Alfvén waves alone heat the corona and accelerate the solar wind. The one-fluid model by Withbroe [1988] which 
is based on earlier stellar models by Hammer [1983, and references therein], assumes that the solar wind is accelerated to high speeds by Alfvén waves, but that there exists an additional unknown energy flux that extends beyond the solar surface and produces temperature maxima at distances $\mathrm{r}>R_{S}$. This model seems to fit observational constraints in the inner corona and in interplanetary space reasonably well. The weakness of this model is that no physical mechanism is attributed to the heating in the inner corona. A detailed discussion of possible underlying mechanism(s) has recently been provided by Axford and McKenzie [1991; see also Axford and McKenzie, this volume, and references therein].

The models described above have their inner boundary at temperatures typical of the middle transition region. It would be desirable to extend these models to lower transition region and even chromospheric temperatures. However, the lower transition region and chromosphere are not optically thin, and modeling the radiative losses of the electrons which are important at these low temperatures, is not an easy task. So far no model exists that connects the chromosphere, transition region and corona in a realistic way.

In the following we present some results of two- and three-fluid models that take into account the heating from transition region to coronal temperatures in an ad hoc way similar to the Withbroe [1988] model.

\section{Two-Fluid Solar Wind Model}

The equations describing the steady solar wind flow, that is continuity, momentum and an energy equation for each of the species (electrons and protons), are solved using a time-dependent solar wind code. This code is described in detail in Habbal et al. [1994]. This model takes into account adiabatic cooling and conduction for both particle species. Provisions for acceleration by Alfvén waves, as well as electron and proton heating, are included in the momentum and energy equations respectively. Radiative losses of the electrons are taken into account in the manner parameterized for an optically thin transition region by Rosner et al. [1978]. The use of a time-dependent code avoids the numerical difficulties encountered in calculating through critical points. For every set of input parameters, the code is run until a steady state is reached, typically after 50,000 iterations.

To reproduce the temperature increase in the inner corona as indicated by observational constraints, we choose a heating function of the form

$$
h=h_{0} e^{-\left(r-r_{1}\right) / \lambda}
$$

where $h_{0}$ is the strength of the heating term in $\operatorname{erg~} \mathrm{cm}^{-3} \mathrm{~s}^{-1}$, and $\mathrm{r}, r_{1}$ and $\lambda$ are in units of solar radius $R_{S}$. The effect of changing the characteristic 
damping length of this unknown heating mechanism was studied in detail by Withbroe [1988]. The same heating function is applied to electrons and protons.

In Figure 1 a high-speed solar wind solution is compared to observational constraints derived from space-based SPARTAN 201-01 and ground based Mauna Loa White Light coronagraph observations [Guhathakurta and Fisher, 1995]. The inner boundary condition of the model computations are: density, $n_{0}=710^{8} \mathrm{~cm}^{-3}$, magnetic field $B_{0}=1.85 \mathrm{G}$, helium abundance defined as the ratio of fully ionized helium to proton densities, $\alpha=0.03=$ const., Alfvén wave velocity amplitude, $\delta v_{0}=20 \mathrm{~km} \mathrm{~s}^{-1}$. The heating function for both electrons and protons is specified by: $h_{0}=410^{-8} \mathrm{erg} \mathrm{cm}^{-3} \mathrm{~s}^{-1}$, $r_{1}=2 R_{S}$, and $\lambda=0.7 R_{S}$. The electron and proton temperatures are set equal at the inner boundary, $T_{e 0}=T_{p 0}=210^{5} \mathrm{~K}$. The flow tube of the solar wind stream is assumed to expand twice as fast as radial. The parameters at $1 \mathrm{AU}$ calculated from the model are: flow speed $v_{E}=764 \mathrm{~km} \mathrm{~s}^{-1}$, density $n_{E}=2.1 \mathrm{~cm}^{-3}$, electron temperature $T_{e E}=2.610^{5} \mathrm{~K}$, and the proton temperature $T_{p E}=2.6510^{5} \mathrm{~K}$. These values are all inside the limits placed by observations on high speed streams. This example which is presented in more detail in Habbal et al. [1995] is the result of thorough comparisons between a large number of model computations where the input parameters were varied over wide parameter ranges, and the presented observations. Chosen here was the best fit to the data obtained from the parameter study. Figure 1a compares the calculated and observed density profiles in the corona out to $6 R_{S}$. Figure 1 b shows the "empirical" velocity profile calculated from the empirical upper and lower limits on the density and the assumption that the mass flux is constant. This example gives significantly different temperatures for the protons and electrons (Figure 1d) in the inner corona. This is consistent with the hydrostatic temperatures, $T_{h}$, derived from the density gradient in Figure 1a under the assumption that $T_{e}=T_{p}$, and that the pressure gradient force balances gravity. Thus $T_{h}$ displayed in Figure 1c, might be regarded as an average over $T_{e}$ and $T_{p}$. Since $T_{h}$ is larger than typical electron temperatures in coronal holes [e.g. Habbal et al., 1993], the proton temperature should be larger than the electron temperature. Obesrvational indications of this temperature differences have previously been reported by Noci and Porri [1983].

Also shown in Figure 1d is the effective temperature defined as [e.g. Esser et al., 1986]

$$
T_{e f f}=T_{p}+C\left(m_{p} / 2 k\right)<\delta v^{2}>
$$

where $m_{p}$ is the proton mass, $\mathrm{k}$ the Boltzmann constant, and $\mathrm{C}$ a constant [Esser et al., 1986]. It is commonly assumed that the width of the resonantly scattered Lyman-alpha profile reflects the effective temperature of the protons. As shown in the figure, this temperature can be expected to be high, 

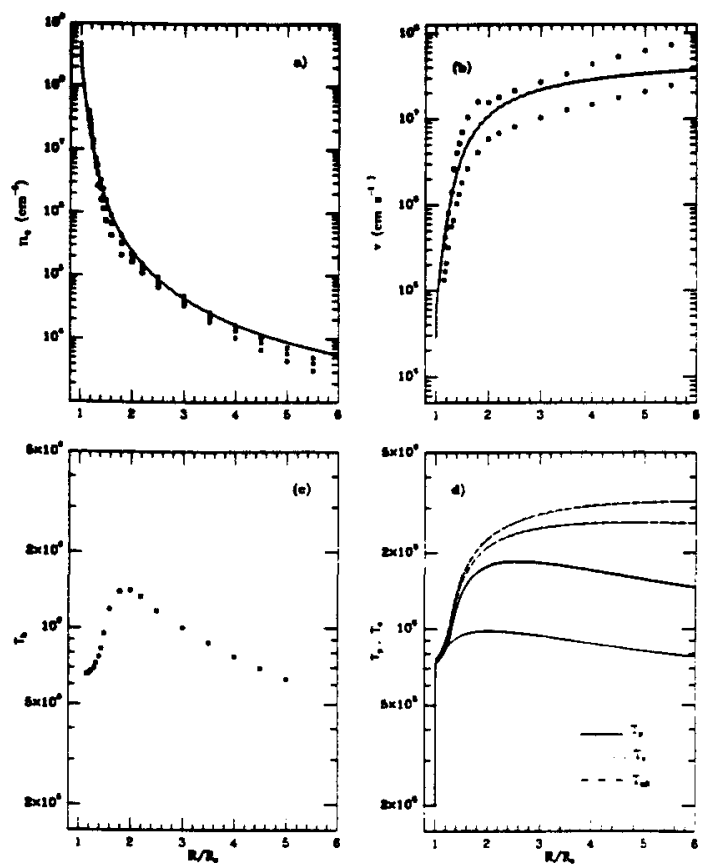

Fig. 1. Composite plot for a coronal hole showing (a) the density profile inferred from White Light data ( $\square$ ) and from the model computation (solid line). The three symbols for the data at each heliocentric distance correspond to the inferred density and upper and lower limits as derived from the uncertainty in the data; (b) data points are for the upper and lower limits of the "empirical" flow speed which are calculated from the upper and lower limits on the empirical density assuming a constant mass flux and a more than radial expansion factor of 2 . The solid line is for the computed flow speed; (c) the hydrostatic temperature calculated from the data, (d) the computed electron temperature, proton temperature, and effective temperature for $\mathrm{C}=1$ and $2 / 3$ (from $\mathrm{Habbal}$ et al. [1995]).

particularly compared to the thermal electron temperature. This has also been confirmed by recent observations of the resonantly scattered Lyman alpha profile [Kohl et al., 1994].

\section{Three-Fluid Solar Wind Model}

In the model outlined in the previous section and described in more detail in Habbal et al. [1994], we now include fully ionized helium by solving an additional continuity, momentum and energy equation for the alpha particles. These equations are essentially the same as the corresponding equations for the protons, except that the proton mass, density, flow speed and charge are replaced by their respective quantities for the helium. The conduction in the helium gas is neglected in the calculations described below. Included in the 

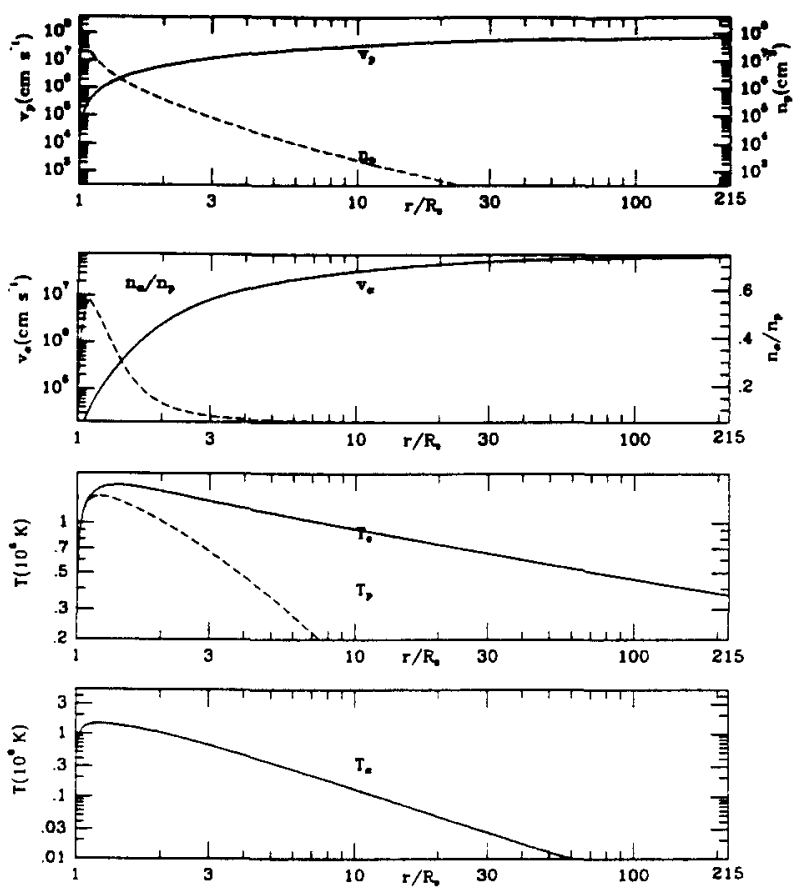

Fig. 2. Example of a high speed stream (a) proton flow speed (solid line) and proton density (dashed line), (b) flow speed of the alpha particles (solid line) and helium abundance (dashed line), (c) and (d) electron, proton and helium temperature.

Table I. Parameter Values for Theoretical Models

\begin{tabular}{ccccccc}
\hline model & figure & $\begin{array}{c}H_{0} \\
\mathrm{erg} \mathrm{cm}^{-3} \mathrm{~s}^{-1}\end{array}$ & $\begin{array}{c}\lambda \\
R_{S}\end{array}$ & $\begin{array}{c}T_{0} \\
10^{6} \mathrm{~K}\end{array}$ & $\begin{array}{c}T_{\max } \\
10^{6} \mathrm{~K}\end{array}$ & $\begin{array}{c}\left\langle\delta v_{0}^{2}\right\rangle^{1 / 2} \\
\mathrm{~km} \mathrm{~s}^{-1}\end{array}$ \\
\hline 1 & 2 & 5.25 & 0.2 & 0.5 & 1.7 & 1.8 \\
2 & $3 \mathrm{a}$ & - & - & 1.4 & - & $10-30$ \\
3 & $3 \mathrm{~b}$ & - & - & 1.8 & - & 10 \\
4 & $3 \mathrm{c} / \mathrm{d}$ & - & - & 1.2 & - & $0-20$ \\
5 & 3c/d & 4.8 & 0.3 & 0.5 & 1.2 & $0-20$
\end{tabular}

calculations shown in Figures 2 and 3 is a heating term in the inner corona of the form given by Equation 1. In this section, however, we only heat the electrons and neglect the heating of the protons and alpha particles.

In Figure 2 we have selected a solution which represents a high speed stream. The density at the coronal base is $n_{0}=510^{8} \mathrm{~cm}^{-3}$, the magnetic field is $B_{0}=1.5 \mathrm{G}$, the geometry is radial. The other input parameters are given in Table 1, case 1. Figure 1a shows the proton flow speed (solid line) and proton density (dashed line) as a function of heliocentric distance. In 
Figure $2 \mathrm{~b}$ we have plotted the flow speed of the alpha particles (solid line) and the helium abundance (dashed line). The helium abundance increases in this case from $10 \%$ at the coronal base to about $56 \%$ at $1.1 R_{S}$ [see also Bürgi, 1992]. The abundance falls then off rather quickly until it reaches an almost constant value of about $5 \%$. Since it is difficult to imagine a mechanism that changes the abundance at larger distances from the Sun, we consider the helium abundance a rather strong constraint on the model, on equal footing with the mass flux. The flow speed of the helium is equal to the flow speed of the protons in this model, in contradiction to the observations in which the flow speed of the alpha particles usually exceeds that of the protons by about the local Alfvén speed. This phenomenon is assumed to be the result of preferential acceleration of the alpha particles by waves [e.g. Isenberg, 1982, and references therein]. In the example shown in Figure 2c and $2 \mathrm{~d}$, the temperature of the alpha particles and protons is lower than the electron temperature in the inner corona since only the electrons are heated in this case. The electron temperature is relatively high about $1.710^{6} \mathrm{~K}$ at $1.4 R_{S}$. Note that there are no observational constraints on the temperatures of the species in this region. At $1 \mathrm{AU}$ the electron temperature is higher than both the proton and alpha particle temperature. In-situ observations in interplanetary space show that the alpha particles are hotter than the protons, and the protons are hotter than the electrons. This temperature difference is usually attributed to preferential heating of the protons and alpha particles [e.g. Isenberg, 1982, and references therein]. This preferential heating and acceleration is assumed to take place at larger distances from the Sun where it will effect the flow speeds and temperatures but will have little effect on the mass flux and helium abundance. The example in Figure 2 shows that a set of input parameters at the coronal base chosen in agreement with observational limits, can result in solutions that meet the most important constraints placed by observations at $1 \mathrm{AU}$, namely mass flux and helium abundance. The parameters of the models that deviate the most from observations, i.e. temperatures and helium flow speed, could be significantly altered between the inner corona and $1 \mathrm{AU}$ due to wave particle interactions for example.

In Figure 3a and 3b we have plotted the proton flux at $1 \mathrm{AU}$ as a function of wave velocity amplitude, $\delta v$, and helium abundance, $n_{\alpha} / n_{p}$. In these calculations we have neglected the heat input in the inner corona. The temperature maximum is therefore the value at the coronal base. The boundary conditions for these cases are given in Table 1, cases 2 and 3. As the two figures demonstrate, the mass flux is decreased by a significant fraction when the helium abundance increases from 1 to $40 \%$ [see also Leer and Holzer, 1991; Bürgi, 1992], and the mass flux is less sensitive to the increase of the wave velocity amplitude at the coronal base. In Figure $3 \mathrm{c}$ and $\mathrm{d}$ we investigate this same effect when the heating in the inner corona is included in 

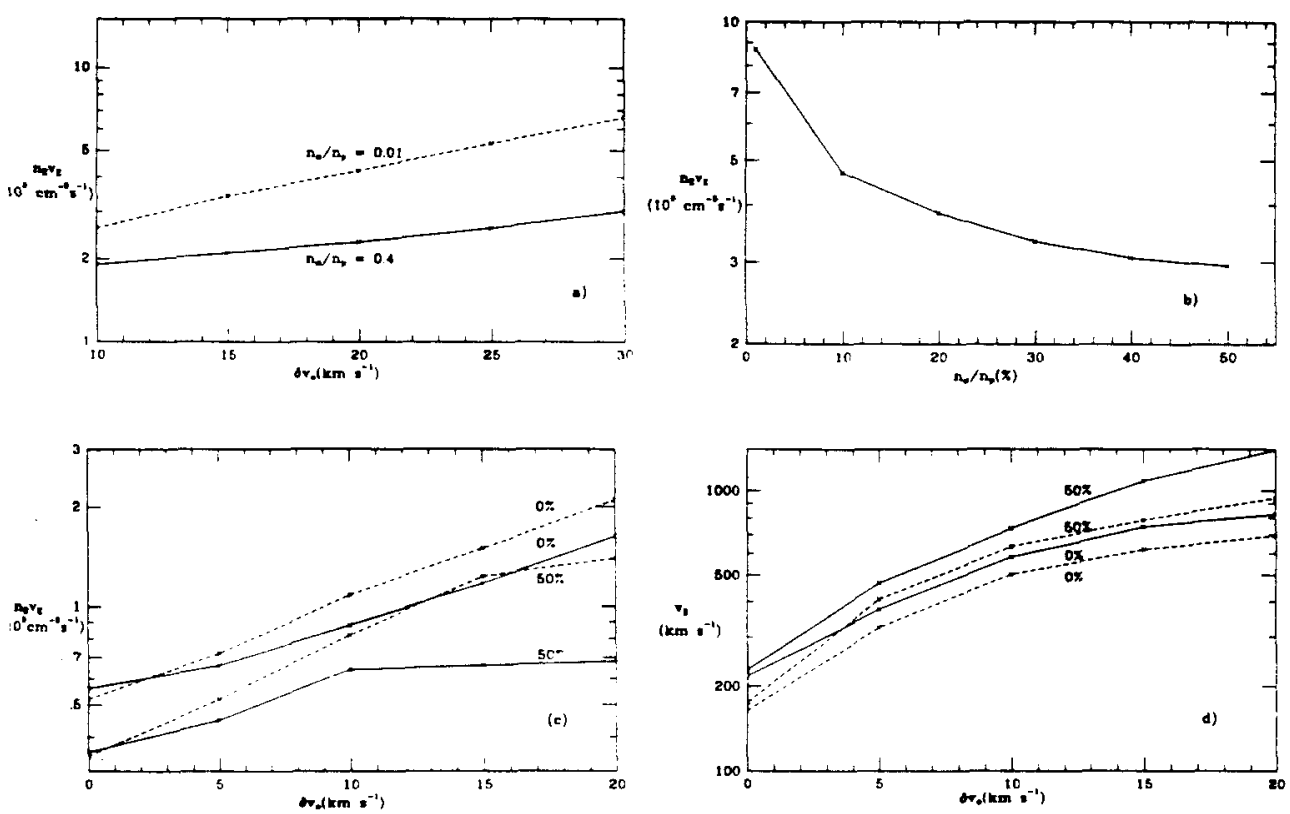

Fig. 3. (a) Proton flux at $1 \mathrm{AU}$ as a function of wave velocity amplitude at the coronal base when the alpha abundance is 1 and $40 \%$. (b) Proton flux as a function of helium abundance at the coronal base. No heating is included in the inner corona in these two cases. (c) Proton flux at $1 \mathrm{AU}$ as a function of wave velocity amplitude at the coronal base for 0 and $50 \%$ helium when heating of the electrons in the inner corona is included (solid lines) and when the heating is neglected (dashed lines), (d) corresponding proton flow speeds at $1 \mathrm{AU}$. The density and magnetic field at the coronal base are $n_{e}=510^{8}$ $\mathrm{cm}^{-3}, B_{0}=1.5 \mathrm{G}$, and the geometry is radial. The other input parameters to the models are given in Table 1.

the model. The input parameters for these models are given in Table 1 cases 4 (dashed lines in the figure) and 5 (solid lines in the figure). Comparing the cases when $n_{\alpha} / n_{p}=0 \%$ shows that moving the maximum temperature from $1 R_{S}$ to about $1.3 R_{S}$ results in a mass flux that is less sensitive to increases in the wave velocity amplitude. This effect is even larger when helium is included in the calculations. Figure $3 \mathrm{~d}$ shows the corresponding flow speeds at $1 \mathrm{AU}$.

\section{Conclusions}

From the few examples presented above, it is clear that including the heating in the inner corona is extremely important since it influences the behavior of the solar wind flow at larger distances from the Sun as originally stated by 
Hollweg [1986] and Withbroe [1988]. The inclusion of helium, and probably other minor ions, seems to be important as well, at least for certain parameter ranges. It is also desirable to extend these models to the lower transition region and chromosphere, but only if the proper radiation balance in these regions is taken into account. To make comparisons between models and in-situ observations more meaningful it seems timely to start a new effort to include preferential heating and acceleration at larger distances from the Sun in the models. Another aspect which has mostly been ignored in the models is the difference between parallel and perpendicular temperatures, as well as the non-Maxwellian velocity distributions in the solar wind.

\section{Acknowledgements}

We thank A. Mossman for the preparation of the figures. Support for this work was provided by NASA grant NAGW-3513 and NAGW-249.

\section{References}

Axford, W. I., 1995, this volume

Axford, W. I., and J. F. McKenzie, 1992 Solar Wind 7, eds. E. Marsch and R. Schwenn, Pergamon: Oxford, p 1

Bürgi, A., 1992, J. Geophys. Res., 97, 3137.

Esser, R., E. Leer, S. R. Habbal, and G. L. Withbroe, 1986, J. Geophys. Res., 91, 2950.

Guhathakurta, M., and R. Fisher, 1995, Astrophys. J. Lett., in press.

Habbal, S. R., R. Esser, and M. Arndt, 1993, Astrophys. J., 413, 435.

Habbal, S. R., Y. Q. Hu, and R. Esser, 1994, J. Geophys. Res., 99, 8465.

Habbal, S. R., R.Esser, M. Guhathakurta, and R. Fisher, 1995, Geophys. Res. Let., in press.

Hammer, R., 1983, Adv. Space Res., 2, 261.

Hollweg, J. V., 1986, J. Geophys. Res., 91, 4111.

Hollweg, J. V., and W. Johnson, 1988, J. Geophys. Res., 93, 9547.

Isenberg, P. A., 1982, Solar Wind Five, ed. M. Neugebauer, NASA Conference Publication 2280, p. 655.

Kohl, J. A., L. D. Gardner, L. Strachan, and D. M. Hassler, 1994, Space. Sci. Rev., 70, 253.

Leer, E., and T. E. Holzer, 1991, Ann. Geophys. Res., 9, 196.

Noci, G., A. Porri, 1983, IAGA $18^{\text {th }}$ Gen. Ass., Hamburg, paper 4L.04.

Parker, E. N., 1958, Astrophys. J., 126, 664.

Parker, E. N., 1963, Interplanetary Dynamical Processes, Interscience: New York.

Rosner, R., W. H. Tucker, and G. S. Vaiana, 1978, Astrophys. J., $220,643$.

Withbroe, G. L., 1988, Astrophys. J., 325, 442. 